\title{
Lapurdum
}

LAPURDUM Euskal ikerketen aldizkaria | Revue d'études basques |

Revista de estudios vascos | Basque studies review

$5 \mid 2000$

Numéro V

\section{Miscellanées basco-ibériques}

Hector Iglesias

\section{OpenEdition \\ Journals}

Édition électronique

URL : http://journals.openedition.org/lapurdum/1302

DOI : 10.4000/lapurdum.1302

ISSN : 1965-0655

Éditeur

IKER

Édition imprimée

Date de publication : 1 octobre 2000

Pagination : 167-180

ISBN : 2-84127-161-7

ISSN : $1273-3830$

\section{Référence électronique}

Hector Iglesias, « Miscellanées basco-ibériques », Lapurdum [En ligne], 5 | 2000, mis en ligne le 01 juin 2009, consulté le 03 juillet 2020. URL : http://journals.openedition.org/lapurdum/1302 ; DOI : https:// doi.org/10.4000/lapurdum.1302 


\title{
Miscellanées basco-ibériques
}

\author{
Hector IGLESIAS
}

h.iglesias@biarritz.fr

Le professeur Jacques Allières nous fit part il $y$ a quelques mois d'une suggestion extrêmement intéressante concernant un patronyme basque très curieux.

Il s'agit d'un patronyme euskarien dont la signification était jusqu'à présent inconnue, si I'on écarte évidemment les étymologies fantaisistes. Luis Michelena dans son ouvrage Apellidos vascos ne I'explique pas, probablement par prudence' ${ }^{\mathbf{1}}$.

Nous faisons référence au nom de famille basque Haritschelhar (et ses variantes graphiques Haritchelar et Haritschelar).

La principale caractéristique de ce nom est qu'il est inexplicable, l'étymologie selon laquelle on aurait eu affaire à un (très) hypothétique et (très) curieux haritz-el(h)ar / haritx-el(h)ar, « (lieu de) chênes pédonculés dans les bruyères » ne constituant rien d'autre qu'une tentative facile d'expliquer l'inexplicable.

Certaines certitudes existaient - et existent - toutefois : il est sûr qu'à l'initiale, on a le basque haritz, «chêne pédonculé », la finale *-chel(h)ar demeurant quant à elle énigmatique. 
C'est à présent qu'intervient le professeur Jacques Allières : celui-ci nous fit part quelques mois avant sa disparition d'une intime conviction qu'il nourrissait depuis quelques temps : *chel(h)ar (en orthographe basque moderne $* x \boldsymbol{x}(\boldsymbol{h}) a \boldsymbol{r})$ ne constitue probablement rien d'autre, pensait-il, qu'une variante palatalisée du très banal zel(h)ai (dont la variante palatalisée serait $\boldsymbol{x e l}(\boldsymbol{h}) \boldsymbol{a i})$.

Notre étonnement fut à la mesure de la clairvoyance dont faisait alors preuve notre interlocuteur : grand. Cette hypothèse de travail ne nous avait en effet jamais traversé l'esprit, pas plus qu'elle n'avait manifestement traversé (après plusieurs vérifications de notre part) celui de plusieurs autres bascologues prestigieux dont, entre autres, Luis Michelena comme nous le verrons par la suite.

Il fallait y penser et le seul à I'avoir fait aura été Jacques Allières.

En effet, *xel(h)ar(r) / *zel(h)ar(r) ne doit et ne peut être rien d'autre que le doublet de zel(h)ai au même titre que ibar(r) est celui de ibai et que bizkar(r) celui de bizkai.

La série ibai $\leftrightarrow \operatorname{ibar}(r) /$ bizkai $\leftrightarrow$ bizkar(r) est la plus connue. Mais il existe d'autres exemples, hormis celui de *zel(h)ar(r) / zel(h)ai qui vient d'être, on I'a vu, découvert par Jacques Allières, on dispose également, semble-t-il, de I'alternance belhar(r) / Belhai (toponyme souletin qu'il ne faut pas confondre avec le vocable baztanais belai qui est probablement une contraction d'un belagai également attesté) $\leftrightarrow$ probablement Belhay (anciennes maisons de Biarritz et Anglet), très probablement uztar( $\boldsymbol{r})$, «joug» uztai, « arc, arceau, cercle », etc.

Antonio Tovar, dont nous reparlerons plus loin, avait déjà remarqué qu'il existait parfois en basque une alternance $\boldsymbol{- i} / \boldsymbol{r} \boldsymbol{r}(\boldsymbol{r})^{\mathbf{2}}$, par exemple dans (h)amar(r), «dix» / amai, «limite 》 (dix représentant évidemment une limite physique puisque nous n'avons que dix doigts) ainsi que le cas du suffixe basque $-\boldsymbol{k} \boldsymbol{k}(\boldsymbol{r})$, « qui a tendance à » (utilisé 
avec les verbes) qui alterne avec -koi, « qui a le vice de » (utilisé avec les substantifs) comme dans hilkor(r), «mortel » / aragikoi, « carnivore », etc. ; mais à aucun moment cependant Antonio Tovar ne semble avoir soupçonné l'existence d'une forme zelhar(r) qui aurait alterné avec zelhai.

La plupart du temps lorsque les deux variantes ont survécu dans le lexique, une des deux a connu une évolution sémantique comme cela est le cas dans la paire ibai, « rivière » / ibar( $\boldsymbol{r})$, « vallon, plaine, vallée ».

Dans d'autres cas, une des variantes a disparu (elle a été éliminée par son doublet) et n'existe plus qu'à l'état fossile dans la toponymie (un des énormes intérêts qu'offrent en effet les formes toponymiques est que ces dernières ont fossilisé plusieurs lexèmes ou proto-formes lexématiques éliminés par la suite du lexique courant).

C'est le cas bien évidemment du terme bizkai qui n'est parvenu à nous que grâce à ce processus appelé «fossilisation toponymique ». Dans le cas de zel(h)ai, c'est l'inverse qui s'est manifestement produit : ce n'est cette dernière forme qui a disparu, mais au contraire la forme $\boldsymbol{x e l}(\boldsymbol{h}) \operatorname{ar}(\boldsymbol{r})$ qui nous est miraculeusement parvenue grâce au patronyme Haritschelhar (on mesure dès lors l'énorme intérêt que présente ce nom basque).

Le nom Haritschelhar ne peut dès lors guère signifier que haritz + *zelhar(r) variante palatalisée de $\operatorname{xel}(\boldsymbol{h}) \operatorname{ar}(\boldsymbol{r})$, « plateau, plaine du ou des chênes » au même titre que Otsozelhaia signifie «la plaine des loups» (il s'agit en réalité d'une forme refaite à partir d'une ancienne variante attestée au Moyen Âge Otsacelhaya avec otsaapparaissant régulièrement en composition à la place de otso $^{\mathbf{3}}$ ).

L'hypothèse de Jacques Allières est extrêmement probable (le doute, si doute il devait y avoir, étant dès lors infime) et c'est d'ailleurs la seule qui puisse être vraiment retenue car elle est logique, cohérente 
et surtout elle s'inscrit dans une série attestée de lexèmes où alternent les suffixes $\boldsymbol{- r}(\boldsymbol{r})$ et $\boldsymbol{- \boldsymbol { i }}$ sans que I'on sache cependant quel était exactement à l'origine le rôle de ces derniers (quelle différence y avait-il en effet à I'origine entre $\boldsymbol{i b a}[\boldsymbol{r}(\boldsymbol{r})]$ et $\boldsymbol{i} \boldsymbol{b} \boldsymbol{a}[\boldsymbol{i}]$ ?).

Cette découverte peut être qualifiée d'importante car, au-delà du fait que I'on dispose d'un nouveau terme ayant appartenu au proto-basque et probablement au basque ancien, elle nous renvoie manifestement à la langue ibère (I'unité de celle-ci ne faisant aujourd'hui guère de doute, si on ne prend pas en compte évidemment le celtibère et le tartessien, cf. Velaza, 1996) ou, si I'on préfère, à la théorie «basco-ibérique ».

En effet, en ibère on dispose de plusieurs inscriptions où apparaît à plusieurs reprises un même lexème (bien identifié) dans des stèles funéraires. Prenons par exemple l'inscription apparaissant dans celle de Cabanes (localité située entre Tarragone et Castellón) :

\section{iltiŕbikisen : seltar : $\overline{\mathbf{m}} i$}

Le syllabaire ibérique ne distinguant pas les occlusives sourdes des sonores (en effet l'usage est de toujours retranscrire les sourdes, même lorsqu'on sait que I'on a affaire à des sonores), on eût pu très bien écrire (certains auteurs le font d'ailleurs, comme Tovar ${ }^{4}$ ) :

\section{ildiŕbigisen : seldar : $\overline{\mathbf{m}} \mathbf{i}$}

Citons également l'inscription :

\section{ośortarbanen siltar (variante de seltar / seldar)}

Il n'est pas utile ici de citer d'autres exemples qui n'apporterait rien à la démonstration (on pourra se reporter pour cela à la bibliographie citée, c'est-à-dire aux Travaux de Jürgen Untermann, Antonio Tovar, Luis Michelena, Javier Velaza, etc.). 
Depuis longtemps, les spécialistes ont en effet la certitude qu'une partie (en règle générale la première partie) de ces inscriptions funéraires est constituée par le nom du défunt suivi d'un génitif dont la forme est -en (identique à la forme du génitif basque) à propos duquel Luis Michelena avait écrit un article remarqué.

Il est en effet acquis depuis des décennies que l'ibère possédait un génitif en -en identique à celui qu'on trouve en basque. Par exemple : IItiŕbikisen / IIdiŕbigisen signifie « de, qui appartient à I/tiŕbikis (le nom du défunt) $\gg$.

Cela étant acquis, les chercheurs se sont alors posés la question, simple et logique, de savoir quelle pouvait être la signification du terme seldar (retranscrit par convention, on l'a dit, seltar où, d'après les spécialistes, $\boldsymbol{- r}$ - représenterait une vibrante forte $\boldsymbol{- r} \boldsymbol{r}$ - en opposition à un autre -ŕ-, également ibère, qui lui représenterait une vibrante faible comme dans iltiŕ > lat. $\boldsymbol{i l}(\boldsymbol{I}) \boldsymbol{i})$. On a alors supposé que ce mot ibère signifiait « tombe » ou quelque chose d'analogue.

Par la suite, on s'est également demandé s'il n'existait pas en basque un terme équivalent. Plusieurs auteurs (pour la plupart non-bascophones) ont alors simplement ouvert un bon dictionnaire de la langue basque, en général celui d'Azkue, et ont essayé de trouver un mot approchant. Cette méthode ou plutôt ce manque de méthode ne pouvait évidemment rien apporter de positif. Ce qui en effet fut le cas.

On trouva cependant le terme basque zeldor, «faix, faisceau, charge », variante de seldor, «pile de bois». On pensa alors que cette dernière signification pouvait bien correspondre avec le sens de «tombe, sépulture », celle-ci pouvant effectivement avoir ou prendre la forme d'une pile, amoncellement ou tas.

En réalité, et à notre connaissance, un seul auteur a su poser la bonne question : Luis Michelena. Dans son article, intitulé «Ibérico -en $\gg$, l'auteur écrivait en effet ${ }^{5}$ : 
« No nos interesa demasiado [dans le cadre de son article] la cuestión de cúal pudiera ser la traducción más exacta de ib. seldar 'cippus, monumentum, uel sim' ».

Il ajoutait en note :

« La correspondencia vasca de seltar [c'est-à-dire seldar] sería *selar o bien *zelar ( 0 , si se prefiere, *sel(h)ar, etc., con aspiración intercalar en algunos dialectos), ya que no están establecidas las correspondencias que unen, siquiera sea en préstamos, ib. $\boldsymbol{s}, \boldsymbol{s}$ y vasc. $\boldsymbol{s}, \boldsymbol{z}$, sibilantes sordas ápico-alveolar y predorsal, respectivamente ».

Il ajoutait cependant :

«Por desgracia, ninguna de estas formas se documenta, que yo sepa, en la lengua [basque] ».

Jacques Allières et Luis Michelena furent, on le voit, deux grands bascologues: I'un, Michelena, avait cherché en vain un terme basque *zel(h)ar(r) (mais il n'avait pas pensé au nom Haritschelhar); I'autre, Allières, I'avait trouvé mais n'avait pas fait le rapprochement avec le terme ibère dont il n'avait probablement pas eu connaissance, comme cela était également jusqu'à il y a encore peu notre cas.

Ce n'est que lorsque nous avons appris l'existence de ce terme ibère (en lisant plusieurs articles, entre autres, de Tovar et Michelena, des travaux la plupart du temps difficile à se procurer et qui auraient malheureusement tendance de nos jours à tomber de plus en plus dans I'oubli), que les dires de Jacques Allières nous revinrent subitement à l'esprit et qu'il nous a ainsi été permis de faire le rapprochement avec la langue ibère.

Jacques Allières aura non seulement su trouver un vieux mot euskarien inusité de nos jours en basque moderne, mais il a fait bien plus, il a découvert une sorte de « chaînon manquant » entre l'ibère d'il y a deux mille ans et le basque actuel, c'est-à-dire l'évolution qui suit : seldar $\rightarrow$ xel(h)ar (variante palatalisée de *zel(h)ar(r) $\rightarrow$ zel(h)ai. 
Du point de vue phonétique, cette évolution est connue des spécialistes. Luis Michelena et la plupart des auteurs, parmi les plus sûrs que la «bascologie » ait connus, renvoient en règle générale à I'article de Von U. Schmoll intitulé «Turma Salluitana. Einige Bemerkungen zur lat. Umschreibung hispanicher Eigennamen » («Turma Salluitana. Quelques remarques sur les transcriptions latines des noms propres espagnols [de I'Antiquité] »).

Voici le fonds de cet article auquel tous les spécialistes versés dans cette difficile question font d'ordinaire référence :

La séquence graphique ibère -/t- apparaît retranscrite en grec $-\boldsymbol{l d}$ (montrant ainsi que dans ce cas on a affaire en ibère à un groupe -ld puisque, on le sait, le syllabaire ibérique ne permet pas de différencier les sourdes des sonores) et en latin -II- ou -I-. C'est pourquoi d'après le fameux document connu sous le nom de Turma Salluitana le nom de l'actuelle ville de Saragosse, apparaissant dans les monnaies ibériques sous la forme saltuie / salduie, est retranscrit en latin Salduuia / Saldubia / Salduba, Pline, 3, 24.

U. Schmoll en avait conclu que le $-\boldsymbol{l d}$ - (-lt-) ibère exprimait, non pas une suite sonore, mais au contraire un seul son que les Grecs et les Romains entendaient comme une sorte de -I- / (-II-) : une sorte d'émission phonique que Rohlfs supposait être un II rétroflexe et analogue à ceux rencontrés en gascon et dans certains parlers hispaniques et italiques, comme il le signalait dans l'un de ses ouvrages $^{6}$ :

« Le traitement très original et tout à fait particulier de l'ancienne géminée en Gascogne nous laisse supposer qu'à l'origine du changement ait dû exister un II suis generis. Déjà Achille Luchaire (AT, p. IX, 1881) avait noté que le phénomène gascon présente une certaine analogie lointaine avec l'évolution de II en Sicile et en Sardaigne, où castellu est devenu castèddu, prononcé avec un $\boldsymbol{d}$ cacuminal. C'est cette idée qui plus tard a été reprise et développé par plusieurs savants: W. Foerster (ZRPh, 1898), Passy (1904), Bourciez, Ronjat 
et par moi-même. Cette singulière prononciation n'est pas limitée en Italie aux deux îles citées. Elle appartient à un large domaine de I'Italie méridionale continentale, à la Corse et encore à certaines zones en Toscane (Rohlfs, GLI § 234). Elle se retrouve en Espagne dans une petite zone des Asturies (à Sistierna), où le 'marteau' est appelé martied'u et où pour 'poule' (esp. gallina) on dit gad'ina ».

Quant à U. Schomll, il écrivait :

«Ich meinte, man könne hier wolh an eine auf ein voridg. Substrat zurückgehende Aussprache denken, obschon 'dem Iberischen und Baskischen kein unmittelbares Indiz fûr retroflexes /I zu entnehmen ist, das dort in der Schreibung vernachlässigt un hier wieder geschwunden sein' könne. Dieses Indiz ist jetzt gefunden : iber. Id (It) bezeichnete ein retroflexes $I(I) \gg$.

En d'autres termes, Shmoll pensait que, bien que le basque et, comme on le croyait en principe, l'ibère ne disposent pas de graphie notant une prononciation rétroflexe, celle-ci aurait cependant été notée en ibère par la séquence graphique Id (It) qui aurait dès lors eu pour fonction principale d'indiquer un II rétroflexe.

\section{L'évolution -Id- > -I- en basque}

En basque la séquence -ld - a effectivement souvent, mais pas toujours, abouti à -II- (non mouillé) puis -I-, à la suite probablement d'une assimilation de $\boldsymbol{d}$ à $\boldsymbol{I}$, les exemples de ce phénomène étant attestés : baldinba- > balimba- ; oroldio > orolio, etc.

Cela expliquerait pourquoi le terme ibère seldar (où $\boldsymbol{s}$ représenterait probablement d'après les spécialistes, c'est du moins une des hypothèses envisagées, un son analogue au $\boldsymbol{z}$ basque, $\mathrm{cf}$. supra) aurait évolué, dans la langue basque cette fois-ci, à zel(h)ar(r) (cf. haritz + zel(h)ar(r) et par la suite alterné avec le moderne zel(h)ai / zelai.

On pourrait ajouter que ce phénomène semblait exister encore au $\mathrm{XVIII}^{\mathrm{e}}$ siècle puisque le nom de la maison d'Anglet appelée Landalde 
devient fréquemment Landalle puis Landale dans la prononciation courante si on en croit les archives notariales consultées. A Bayonne le patronyme Lissalde apparaît couramment écrit Lissalle.

En outre, nous avions déjà, au cours d'un autre article, mentionné un autre fait surprenant : une montagne des Asturies (2519 m.) a pour nom Urriellu avec, sauf erreur, II «mouillé »(en espagnol Urriello). Or, ce nom ressemble étrangement au toponyme alavais Urrialdo, 1025, Urrialdu, 1702, village dont la dernière maison disparut en 1937 à la suite d'un incendie d'après López de Guereñu. En effet, une évolution Urrialdo $>$ *Urriallo $>$ Urriello $>$ Urriellu (avec par la suite $\boldsymbol{I I}$ « mouillé ») est très vraisemblable.

On voit bien à travers cette démonstration que le passage de l'ibère seldar au basque *zelhar(r) / xelhar(r) (cf. Haritschelhar(r)) n'entraîne dans son sillage, du point de vue phonétique, aucun problème insurmontable, voire quelque invraisemblance. Bien au contraire, tout semble concorder.

Etudions à présent la question du point de vue de la signification de l'actuel mot basque zel(h)ai (et de sa variante *zel(h)ar(r) / xel(h)ar(r) découverte par Jacques Allières). Utilisons pour cela les deux dictionnaires les plus sûrs en la matière : celui d'Azkue et celui, en ce qui concerne le Pays Basque Nord, de Lhande.

Azkue : $\ll 1^{\circ}$. champ $-2^{\circ}$. pré. Ce mot par lui-même est plutôt un endroit plat qu'un champ ou un pré, et il s'emploie [aussi] comme adjectif $-3^{\circ}$. sol $-4^{\circ}$. à plat ventre $($ sic $)-5^{\circ}$. plaza, place ».

Lhande: $\ll 1^{\circ}$. plateau $-2^{\circ}$. terre planière $-3^{\circ}$. champ labouré, cultivé $-4^{\circ}$. sol, terre, parquet $\gg$.

C'est évidemment le sens de «à plat ventre » cité par Azkue qui est le plus éloquent: sorura zelai adore baga jausia nago, 'je suis 
tombé à plat ventre sur le sol sans souffle" », le sens de «sol, terre, parquet » donné par Lhande étant également très significatif.

En effet, ce qui caractérise principalement un mort est, on en conviendra aisément, qu'il se trouve à plat ventre $\rightarrow$ eusk. zelai (ou bien sur le dos) et à même le sol, la terre, le parquet.

Or, si on admet que le basque zel(h)ai a connu une variante *zel(h)ar(r) - au même titre que $\boldsymbol{i b a r}(\boldsymbol{r}) / \boldsymbol{i b a i}$, etc. - et que cette variante se rattache elle-même à un prototype *zeldar( $\boldsymbol{r}$ ) que I'on retrouverait dans le mot ibère seldar apparaissant uniquement dans des stèles funéraires, on perçoit dès lors immédiatement les nouvelles perspectives qui s'ouvrent de facto à tous les chercheurs qui s'intéressent à ces questions «basco-ibériques ».

Le terme ibère seldar (toujours, on l'a dit, précédé du nom du défunt si on en croit tous les spécialistes qui se sont penchés sur la question) devait donc - s'il est à l'origine de l'actuel mot basque zelhai - signifier «sol, parquet», c'est-à-dire un endroit où un individu serait couché à même le sol, autrement dit un endroit où serait placé un mort.

Le mot ibère seldar devait donc signifier « sépulture, fosse, tombe, tombeau ». Plusieurs auteurs avaient déjà, on I'a vu, soupçonné cette signification. Cependant, ils ne l'avaient pas fait à la suite d'un raisonnement structuré, mais uniquement par déduction logique. En effet, étant donné que ce terme apparaît toujours dans des inscriptions de caractère funéraire, on en a déduit que le mot signifier «tombe » ou quelque chose d'analogue.

C'était une hypothèse séduisante, mais pas totalement convaincante en partie à cause de la méthode utilisée.

En revanche, ici il ne s'agit pas d'une simple déduction logique puisqu'on part du principe que ce terme ibère était également un terme 
du proto-basque (c'est-à-dire que dans ce cas bien précis le basque et l'ibère était une seule et même chose) et qu'il a connu une évolution qui I'a conduit, après une étape en basque ancien en $* z e l(h) a r(r) /$ $\boldsymbol{x e l}(\boldsymbol{h}) \boldsymbol{a r}(\boldsymbol{r})$, à la forme basque moderne zel(h)ai (alternance $-\boldsymbol{r}(\boldsymbol{r}) /$ -i également attestée, notons-le également, en ibère d'après Antonio $\left.\operatorname{Tovar}^{8}\right)$.

En conclusion, ce qui donne de la force à cette hypothèse est :

1) L'existence de nom Haritschelhar(r) < *haritz-zel(h)ar(r), une sorte, on l'a vu, de « chaînon manquant » permettant de relier ici l'ibère au basque ou, si l'on préfère, l'inverse.

2) Une évolution phonétique $-\boldsymbol{I} \boldsymbol{d}-\mathbf{>} \boldsymbol{- I}(\boldsymbol{h})$ - attestée et bien connue des spécialistes (cf. supra).

3) L'existence encore de nos jours en biscaïen du terme zelai au sens de «à plat ventre», paraissant confirmer que son doublet *zel(h)ar(r) découvert par Jacques Allières est effectivement issu, à la suite de l'évolution décrite ci-dessus, d'un prototype basque *zeldar( $\boldsymbol{r}$ ) présente dans l'ibère seldar au sens de «tombe, sépulture » puisque «tombe » / «à plat ventre » sont deux acceptions qui se rejoignent étant donné que toutes les langues - langue basque incluse - évoluent non seulement du point de vue phonétique mais également du point de vue sémantique : par exemple le mot français bureau désignait primitivement une étoffe de bure utilisée la plupart du temps pour recouvrir une table. Puis, à la suite d'une synecdoque le terme a fini par désigner le meuble lui-même, puis par une seconde synecdoque, c'est la pièce contenant ce meuble qui a été ainsi dénommée.

Autrement dit, il est clair que même si l'on venait à connaître la signification exacte d'un vocable ibère ayant existé il y deux mille ans (grâce par exemple à une inscription bilingue), voire celle d'un mot appartenant au proto-basque, et que dans le même temps on 
parvenait à disposer de son résultat, à la suite d'une évolution phonétique ultérieure, en basque moderne, il presque sûr qu'on serait également en présence d'une évolution sémantique.

C'est pourquoi le mot basque zel(h)ai / *zel(h)ar(r) / $\boldsymbol{x e l}(\boldsymbol{h}) \operatorname{ar}(\boldsymbol{r})$, bien que manifestement issu, d'après notre hypothèse, de l'ibère seldar, «tombe » (signification extrêmement probable), ne signifie plus et cela depuis plusieurs siècles «tombeau, fosse, sépulture », mais au contraire «plateau, sol, parquet, à plat ventre », etc. car l'existence d'une évolution sémantique nous paraît dans le cas présent difficilement contestable (puisqu'il est en effet impossible de nier que la plupart des termes basques connaissent eux aussi à un moment ou à un autre une évolution sémantique).

On le voit cette découverte, due principalement à Jacques Allières, est importante car elle permet de rapprocher pour la première fois un mot ibère d'un mot basque d'un point de vue à la fois phonétique et sémantique.

Les seuls mots que I'on avait pu jusqu'à présent rapprocher le plus sûrement du basque étaient, entre autres, il(I)i / iltiŕ / ildiŕ et, dans une moindre mesure, le terme ibère gudua / kutua (mais ce dernier rapprochement offre toute une série de difficulté que nous ne pouvons pas aborder ici).

Il en existe également quelques autres qu'il n'est pas non plus utile de citer dans le cadre de cet article.

Cependant, tous ces rapprochements ne constituaient rien d'autre que des comparaisons concernant la forme des mots et il s'agissait de comparaisons établies à la suite d'une déduction logique où les faits ayant trait à l'évolution phonétique et sémantique des termes en question n'intervenaient guère ou à peine, ce qui n'est pas, on en conviendra, le cas dans le présent article. 


\section{Esquisse de conclusion}

Il est vrai que l'établissement de l'existence d'un mot ibère, qui après avoir connu une évolution, à la fois phonétique et sémantique, continue à exister de nos jours en basque constitue un fait tout à fait remarquable et inattendu.

On ne peut évidemment en tirer la conclusion définitive que les Basques constituent un reliquat de populations ibériques qui ont survécu, pour des raisons mal connues, à la romanisation, probablement des facteurs d'ordre politique, bien que, en ce qui nous concerne, nous en soyons à peu près convaincus : les Basques doivent et ne peuvent être finalement que les descendants des populations pré-indo-européennes d'origine ibérique, quoique, dans l'actuel état de nos connaissances, cela ne puisse être, il est vrai, formellement prouvé.

Pour conclure, on serait presque tenté, si I'on pouvait se permettre cette expression, de qualifier le patronyme Haritschelhar (< *Haritzelhar $(r)<*$ Haritzeldar $(r)<*$ haritz + zeldar $(r))$, un nom basque, on I'aura compris, extrêmement ancien, surprenant et intéressant, de nom en partie « basco-ibère ».

\section{Le toponyme historique Ilbar(r)itz}

(quartier historique de Bidart et aujourd'hui également plage et quartier de Biarritz: Libarritz, 1644, Ilbarritz, 1761, Ilbarits, Cassini, Ilbarritz, 1774, Illibarits en 1826 d'après les «Ingénieurs Hydrographes de la Marine», Libarrits, 1845, Ilbarrits, 1863, aujourd'hui « Ilbarritz »).

Si nous mentionnons à présent ce toponyme historique de la Côte Basque, c'est principalement pour l'intérêt scientifique qu'il paraît représenter, en particulier en ce qui concerne les Etudes Basques, car d'une part non seulement aucun spécialiste en toponomastique n'a 
jamais pu réussir à l'expliquer mais d'autre part de par sa structure même, nous avons de grandes raisons de croire qu'il s'agit d'un nom ancien, c'est-à-dire créé probablement entre le début de notre ère et le $\mathrm{XI}^{\mathrm{e}}$ siècle.

La plus ancienne mention attestée de ce nom a été découverte à la suite de plusieurs recherches que nous avions entreprises il y a quelques années parmi les archives de la ville de Biarritz.

Le nom apparaît en effet dans un document de 1644 sous la forme Libarritz, une forme qui curieusement réapparaît sous une graphie identique, à l'exception de la finale, dans un acte notarié de 1845, c'est-à-dire Libarrits, la forme la plus intéressante, quoique suspecte pour des raisons que nous allons aborder à présent, étant cependant celle donnée en 1826 par les ingénieurs hydrographes de la Marine, c'est-à-dire Illibarits.

Il se peut que les formes Ilbarritz / Ilbar(r)its soient secondaires, c'est-à-dire issues justement d'une plus ancienne forme Libarritz à la suite d'une métathèse, probablement récente, c'est-à-dire post-médiévale, $\boldsymbol{L i}$ - > II-.

En Navarre il semblerait qu'on rencontre I'alternance Lizarbe (patronyme) et Ilzarbe (nom de village: Ilçarue, 1242, I/çarbe, 1279), une alternance qui rappelle étrangement I'alternance Libarritz / Ilbarritz.

Jacques Allières ${ }^{9}$ explique I'apparition de l'initiale $\mathbf{L i}$ - ainsi : le - Isimple intervocalique passant normalement à $\boldsymbol{- r}$ - en basque (ili $>\boldsymbol{i} \boldsymbol{i} \boldsymbol{i}$, Velasco > Berasko, Galindo > Garindo), il est dès lors probable que Libarritz est issue d'une ancienne forme *Ilibarr- où la voyelle initiale $\boldsymbol{i}$ - serait tombée avant que le passage de $\boldsymbol{- \boldsymbol { l }}$ - à $\boldsymbol{- r}$ - ne se produise, ce qui expliquerait la conservation du $\boldsymbol{I -}$ initial dans Libarritz ${ }^{10}$. 
Or, étant donné qu'en basque le changement $-\boldsymbol{I}-\boldsymbol{>} \boldsymbol{- r}$ - semble antérieur au $\mathrm{XI}^{\mathrm{e}}$ siècle (Borinivar, 1025 < molinu, actuel Bolívar, Alava), cela prouverait l'ancienneté du nom puisque le changement *ilibarr- > libarr- aurait nécessairement dû avoir lieu avant cette époque.

Il existe en effet plusieurs autres exemples qui donnent du poids à cette hypothèse :

a) Les deux noms navarrais Mendilibarri (Valdega ou vallée d'Ega, Estella) et Mongiliberri (vallée de Yerri) où à l'intérieur du nom apparaissent d'après Achille Luchaire, à la suite manifestement d'une fossilisation toponymique, les formes -ilibarri / -iliberri.

b) Encore plus intéressant : le village navarrais de Liberri (vallée de Longuida, Sangüesa), autrefois Liuerri, 1174, Liverri, 1268, Liuerre, 1278 que plusieurs spécialistes pensent issu d'un ancien *Iliberri. Or, il existait autrefois en Navarre un village, aujourd'hui abandonné, dans la vallée d'Esteribar dont le nom est documenté au Moyen Âge sous la forme Iriberri (donc elle même issue nécessairement d'une forme plus ancienne *Iliberri) qui apparaît également dans la documentation médiévale sous la forme Liberri ${ }^{\mathbf{1 1}}$ («illum monasterium quod habeo in Ezteribar et nominatur Liberri », 1066), ce qui permet de supposer que *Iliberri a pour résultat, soit Iriberri, soit Liberri, la forme esteribarraise Iriberri étant ici probablement une forme populaire, les bascophones de la région sachant intuitivement, lorsqu'ils parlaient en basque, que la forme Liberri ne pouvait être rien d'autre qu'une variante du très banal et populaire Iriberri.

En revanche, dans la vallée de Longuida, relevant du bailliage de Sangüesa, débasquisé depuis très longtemps, donc dépourvu de tradition orale bascophone ancienne, il n'y a pas lieu de s'étonner que seule la forme Liberri (avec $\boldsymbol{L}$ - à l'initiale) soit documentée depuis le 
$\mathrm{XII}^{\mathrm{e}}$ siècle car à l'époque la chute, nécessairement antérieure au $\mathrm{XI}^{\mathrm{e}}$ siècle, du $\boldsymbol{i}$ - initial que Schuchardt identifiait à un préfixe que I'on rencontrerait également en berbère ${ }^{\mathbf{1 2}}$ - ce qui expliquerait d'après Jacques Allières la conservation du $\boldsymbol{L}$ - initial qui ne pouvait plus désormais passer à -r- - avait déjà eu lieu, la non-existence de bascophones dans la région ne permettant plus par la suite le rétablissement d'une forme populaire Iriberri comme dans le cas de l'Iriberri de la vallée d'Esteribar.

Il est donc probable, comme le pensait au demeurant Menéndez Pidal ${ }^{\mathbf{1 3}}$, que cet Liberri navarrais est issu d'un plus ancien $*$ Iliberri. En conséquence, il est également possible que le nom labourdin Ilbarritz / Libarritz soit issu d'un ancien Ilibarritz (sans astérisque car un Illibarits est effectivement, on I'a vu, attesté, quoique suspect pour les raisons évoquées ci-dessus).

C'est à partir de maintenant que la question prend un tour des plus intéressants: René Lafon, l'éminent bascologue, avait remarqué, en faisant preuve d'une grande intuition et érudition, qu'il exitait en Andalousie, dans la municipalité de Montejaque, près de Ronda (province de Málaga), un caserío appelé Libar et une sierra du même nom : Sierra de Libar (prononcé manifestement Libarr en espagnol), le nom n'étant explicable ni par le latin ni par l'arabe ${ }^{\mathbf{1 4}}$.

Cet auteur comparait ce nom avec celui de Libarona, quartier de Gatica, village situé au nord de Bilbao, c'est-à-dire Libar + -ona ${ }^{15}$ ainsi que celui de Libaros $^{\mathbf{1 6}}$, c'est-à-dire Libar + -os, commune des Hautes-Pyrénées, à 32 kilomètres à l'est de Tarbes, par conséquent dans I'ancienne Aquitania de Jules César, autrefois Livarossio, XIV siècle.

On pourrait ajouter à cette liste, outre évidemment le toponyme labourdin Libar(r)its / Libarritz, c'est-à-dire Libar(r) + -itz, le nom 
souletin Livarrenx, autrefois Livaren, 1305, Livarren, 1327, Libarren, 1383, c'est-à-dire Libar(r) + -en.

On peut dès lors reconstruire la série toponymique, tout à fait vraisemblable, qui suit: Libar(r) (Andalousie, sans suffixe) / Libar(r)-itz (Labourd) / Libar(r)-en (Soule) / Libar-os (Hautes-Pyrénées) / Libar-ona (Biscaye).

Or, si l'on admet que d'une part « es indiscutible la identidad de vasc[o] Iriberri con el ant[iguo] hisp[ano] Iliberri(s) $\gg^{\mathbf{1 7}}$ et que d'autre part l'évolution Iliberri > Iriberri a également été à un moment donné en concurrence avec l'évolution Iliberri > Liberri, on pourrait approfondir la réflexion de René Lafon en avançant I'hypothése suivante : le nom basque $\operatorname{Iribar}(\boldsymbol{r})$ (et sa variante $\operatorname{Uribar}(\boldsymbol{r})$ ) doit nécessairement être issu d'un ancien *Ilibar(r) - ou *Ulibar( $\boldsymbol{r})$ pour $\operatorname{Uribar}(\boldsymbol{r})$-, c'est-à-dire qu'on aurait eu à un moment donné *Ilibar(r) > Iribar( $\boldsymbol{r})$, ce qui implique qu'un ancien *Ilibar aurait également pu aboutir à une forme $\operatorname{Libar}(\boldsymbol{r})$, celle-ci étant à *Ilibar ce que Liberri est à Iliberri.

En résumé, il est possible que les formes Libar(r) / Libar(r)itz / Libaros / Libar(r)en / Libarona soient issues respectivement de *Ili-bar(r) / *Ili-bar(r)-itz / *Ili-bar(r)-en / *Ili-bar[r]-os ${ }^{18}$ / *Ili-bar $[\boldsymbol{r}]$-ona, dans ces deux dernières la vibrante faible étant peut-être la conséquence d'une erreur, ce genre de confusion entre les vibrantes simples et fortes se produisant fréquemment.

En outre, I'existence du toponyme andalou Libar( $\boldsymbol{r}$ ) concerne la question du «basco-ibérisme » et vient s'ajouter à une longue liste de similitudes toponymiques basco-ibériques extrêmement troublantes ${ }^{\mathbf{1 9}}$. 


\section{Abréviations}

$\begin{array}{lll}\text { ASJU } & = & \text { Anuario del Seminario de Filología Vasca 'Julio de Urquijo' } \\ \text { BBMP } & = & \text { Boletín de la Biblioteca 'Menéndez Pelayo' de Santander } \\ \text { BMB } & = & \text { Bulletin du Musée Basque } \\ \text { BRAH } & = & \text { Boletín de la Real Academia de la Historia } \\ \text { BRSVAP } & = & \text { Boletín de la Real Sociedad Vascongada de los Amigos del País } \\ \text { BSSLAP } & = & \text { Bulletin de la Société des Sciences, Lettres et Arts de Pau } \\ \text { BPH } & =\text { Bulletin Philologique et Historique } \\ \text { EAA } & =\text { Estudios de Arqueología Alavesa } \\ \text { FLV } & =\text { Fontes Linguae Vasconum } \\ \text { RGPSO } & =\text { Revue Géographique des Pyrénées du Sud-Ouest } \\ \boldsymbol{R E A} & =\text { Revue des Etudes Anciennes } \\ \boldsymbol{R I E B} & =\text { Revue Internationale des Etudes Basques } \\ \boldsymbol{R I O} & =\text { Revue Internationale d'Onomastique } \\ \boldsymbol{R} \boldsymbol{R} & =\text { Revista Valenciana de Filología }\end{array}$




\section{Bibliographie}

AGUD, M. \& TOVAR, A., 1989-1993, « Diccionario etimológico vasco », ASJU, T. I-VII, Saint-Sébastien.

ALBERTOS, Ma Lourdes, 1972, «Los nombres éuscaros de las inscripciones hispano-romanas y un Ibarra entre los vettones », EEA, V, pp. 213-218.

ALLIÈRES, J., 1995b, « De I'aquitain au basque », in La langue basque parmi les autres, Actes du colloque de I'URA 1055 du CNRS, Izpegi, Saint-Etienne-de-Baïgorry, pp. 59-70.

ANDERSSON, L., 1971, «Iliberri y la cuestión Vasco-Ibérica », FLV, n० 8, pp. 107-118.

AZKUE, R.-M., 1905-1906, rééd. 1984, Diccionario Vasco-español-francés, Bilbao.

BELTRÁN, P., 1953, «Los textos ibéricos de Liria », RVF, III, 1-4, pp. 36-186.

BOSCH-GIMPERA, P., 1922, «Ensayo de una reconstrucción de la etnología prehistórica de la Península Ibérica », BBMP, IV, pp. 11-50 ; 104-137; 227-281.

BOSCH-GIMPERA, P., 1923, «El problema etnológico vasco y la arqueología », RIEB, XIV, pp. 589-660.

BOSCH-GIMPERA, P., 1932, Etnología de la Península Ibérica, Barcelone.

BOSCH-GIMPERA, P., 1945, El poblamiento antiguo y la formación de los pueblos de España, Mexico.

CARO BAROJA, J., 1988, [1942, 1943], « Observaciones sobre la hipótesis del vasco-iberismo considerada desde el punto de vista histórico », Boletín Emerita, T. X, 2º., pp. 236-286, 1942 et T. XI, 1º, 
pp. 1-59, 1943, apparaissant également in Sobre la lengua vasca $\boldsymbol{y}$ el vasco-iberismo, Estudios Vascos, IX, Txertoa, $3^{\mathrm{e}}$ éd., 1988.

CERRO, 1993, El origen ibérico de la lengua vasca (según los primeros testimonios escritos en lengua ibérica de Andalucía, Aragón, Cataluña, Valencia y Portugal), Ed. Aguaclara, Alicante.

COROMINES, J., 1965, «La toponymie hispanique préromane et la survivance du basque jusqu'au bas moyen âge. Phénomènes de bilinguisme dans les Pyrénées Centrales », Actes et Mémoires du IV Congrès International de Sciences Onomastiques, München, 1960, pp. 105-146. Nous utilisons la nouvelle version revue et corrigée publiée ultérieurement in Estudis de Toponímia Catalana, T. I, Barcelona, 1965, «La survivance du basque jusqu'au bas moyen âge... », pp. 93-151.

COROMINES, J., 1972, Tópica Hespérica. Estudios sobre los antiguos dialectos, el substrato y la toponimia romances, Madrid. DÍEZ MELCÓN, G., 1957, Apellidos castellano-leonenses (siglos IX-XIII, ambos inclusive), Universidad de Granada.

DUBALEN, P., 1914, « Tombes aquitaniques », REA, pp. 217-218.

GATTI, G., 1908, «Lamina de bronzo con iscrizione riferibile alla guerra dei socii italici », pp. 169-226.

GAVEL, H., 1931, « Le problème basque », RGPSO, pp. 222-230.

GÓMEZ-MORENO, M., 1922, «De epigrafía ibérica: el plomo de Alcoy », RFE, pp. 341-353.

GÓMEZ-MORENO, M., 1925, «Sobre los iberos y su lengua », Homenaje ofrecido a Menéndez Pidal, III, pp. 225-240.

GÓMEZ-MORENO, M., 1943, «La escritura ibérica y su lenguaje », BRAH, 251 et suiv. 
GORROCHATEGUI CHURRUCA, J., 1984, Estudio sobre la onomástica indígena de Aquitania, Université du Pays Basque (UPV-EHU), Bilbao.

GUITER, H., 1976, «Onomastique et contacts de langues : exemples des confins pyrénéo-méditerranéens », Onoma, 20/1, pp. 106-127.

HUMBOLDT, W. von, 1866, [1921], Prüfung der Untersucheungen über die Urbewohner Hispaniens vermittelst der Vaskischen Sprache, Impr. F. Dümmler, Berlin, 1821. Version française : Recherches sur les primitifs de l'Espagne à l'aide de la langue basque, Traduction de M. A. Marrast, Paris, impr. Tolnon, 1866.

IGLESIAS, H., 1998, «Sur quelques similitudes toponymiques galaïco-basques et le problème que posent certaines d'entre elles », Lapurdum III, pp. 1-29.

IGLESIAS, H., 1999, « Affinités toponymiques cantabro-pyrénéennes et énigmes historiques », Lapurdum IV, pp. 123-166.

IGLESIAS, H., 2000, «L'inscription ibérique de San Miguel de Liria et le basco-ibérisme en général », $\boldsymbol{F} \boldsymbol{L} \boldsymbol{V}$ 83, pp. 7-27.

IRIGOYEN, A., 1986, En torno a la toponimia vasca $y$ circumpirenaica, Deusto.

LAFON, R., 1999, [1952], « Les écritures anciennes en usage dans la péninsule ibérique d'après les travaux récents », Bulletin Hispanique, LIV, 1952, pp. 165-183 et in Vasconiana, Iker-11, 1999, 57-74.

LAFON, R., 1956a, «Pour l'étude de la langue aquitaine », Actes du deuxième Congrès International d'Etudes Pyrénéennes, Toulouse, pp. 53-63.

LAFON, R., 1956b, « Protohistoire des Landes. Les inscriptions en caractères ibères d'Aubagnan et les inscriptions latines d'Aire-sur-I'Adour », Landes de Gascogne et Chalosse. Actes du 
IX Congrès d'Etudes Régionales tenu à Saint-Sever les 28 et 29 avril 1956 in Fédération historique du Sud-Ouest, Société de Borda, pp. 5-10.

LAFON, R., « Sur la langue des Aquitains et celle des Vascons », BPH, 1957, pp. 1-8.

LAFON, R., 1958, « Noms de lieux d'aspect basque en Andalousie », tiré à part des Actes et Mémoires du $5^{\mathbf{e}}$ Congrès International de Sciences Onomastiques, vol. II, Salamanque, p. 3-32.

LAFON, R., 1965b, « Noms de lieux et noms de personnes basques et ibères : état actuel des problèmes $\gg, \boldsymbol{R I O}, 17^{\mathrm{e}}$ année, pp. 81-92.

LUCHAIRE, A., 1876, «Les origines linguistiques de l'Aquitaine », BSSLAP, pp. 349-423.

MELA, P., 1935, De chorographia, édité par K. Frick, Leipzig.

MENÉNDEZ PIDAL, R., 1968, Toponimia Prerrománica Hispánica, Gredos, Madrid.

MICHELENA, L., 1961, «Comentarios en torno a la lengua ibérica », Zephyrus 12, pp. 5-23.

MICHELENA, L., 1964, Sobre el pasado de la lengua vasca, Collección Auñamendi, Saint-Sébastien.

MICHELENA, L., 1976, «La langue ibère », Actas del II Coloquio sobre lenguas $\boldsymbol{y}$ culturas prerromanas de la Península Ibérica, Tübingen, 17-19 juin, Salamanque, 1979, pp. 23-39.

OLANO SILVA, V., 1945, 1949, 1954, « Toponimia gallega », RDTP, I., 1945, pp. 653-666 ; V., 1949, pp. 626-662 ; X., pp. 190-226.

OROZ ARIZCUREN, F. J., 1976, «El ibérico, lengua en contacto », $\boldsymbol{F} \boldsymbol{L} \boldsymbol{V}$, $n^{\circ}$ 23, pp. 183-194. 
OROZ ARIZCUREN, F. J., 1981, « La relación entre el vasco y el ibérico desde el punto de vista de la teoría del sustrato», Iker-1, pp. 241-255.

OROZ ARIZCUREN, F. J., 1982, « Nuevo topónimo de aspecto vasco en la Bética », ASFJU, XVI, pp. 117-129.

ORPUSTAN, J.-B., 1990, Toponymie basque, Presses Universitaires de Bordeaux.

ORPUSTAN, J.-B., 1999, La langue basque au Moyen Âge, Ed. Izpegi.

PLINE L'ANCIEN, 1850-1851, Histoire Naturelle de Pline, avec la traduction en français, par M. Emile Littré, Paris, 2 vol.

PLINI SECUNDI, C., 1906, Naturalis Historiae, vol. I, Libri I-VI, post Lvdovici Iani obitum, recognovit et scriptvrae discrepantia adiecta edidit Carolvs Mayhoff, MCMVI, Lipsiae in aedibus B. G. Tevbneri. Edition de Carl Mayhoff, qui a repris et renouvelé I'oeuvre de Jan après la mort de celui-ci, Leipzig, Teubner ; version latine seule et pas d'index.

PLINE L'ANCIEN, 1998, Histoire Naturelle, Livre III, texte établi, traduit et commenté par Hubert Zehnacker, Ed. Les Belles Lettres, Paris.

PTOLÉMÉE, 1932, Géographie, édité par E. L. Stevenson, Geography of Claudius Ptolemy, New York (traduction seule).

SCHUCHARDT, H., 1907, «Iberische Deklination », Sitzungsberichte der Wiener Akademie, 157 (II), pp. 1-90.

STRABON, 1966, Géographie, T. II, Liv. III-IV, texte établi et traduit par François Lasserre, Paris.

TOVAR, A., 1959, El euskera y sus parientes, Madrid. 
UNTERMANN, J., 1961, Sprachräume und Sprachbewegungen in vorrömischen Hispanien, Wiesbaden.

UHLENBECK, C., C., 1908, «La declinación ibérica », RIEB, II, pp. 399-409.

UHLENBECK, C., C., 1909, «Contribution à une phonétique comparative des dialectes basques », RIEB, III, pp. 465-503; 1910, pp. 65-118. Traduit en français par $G$. Lacombe.

VELAZA FRÍAS, J., 1996, Epigrafía y lengua ibéricas, Cuadernos de Historia, Madrid.

VERD, G. M., 1980, « Sobre la cuestión vascoibérica», ASJU, XIV, pp. 101-133. 


\section{Notes}

1 Michelena écrit simplement (p. 117, § 381) : «la(h)ar, lar, "zarza" (...) También Haritschelhar, hacia 1225 (sic) Lope de Arachilar de Oteiça (Irache), de difícil análisis ». Ici il semblerait qu'il y ait une erreur de Michelena car la forme Arachilar (forme qui apparaît dans un document du Moyen Âge provenant du monastère navarrais d'Irache : Debebat dona Oria Azenariz iurare in Sancta Mari de Yrax propter domos de Arachilar ; Quod habebant inter se de supra Lope Arachilar de Oteiça, José María Lacarra : c. d. Irache, vol. 0, p. 144, année : 1122-1131) qu'il cite n'est pas et ne peut pas être la forme ancienne du toponyme souletin Haritschelhar, nom de deux maisons de la province de Soule: il s'agit en effet d'un nom, probablement très archaïque, porté par deux maisons de cette province, l'une de ces maisons, dont le nom apparaît écrit d'après I'IGN Haritchelar, étant située dans le village souletin appelée Ligi-Atherei, fr. Licq-Athérey, et I'autre, dont le nom apparaît écrit en revanche Haritchelhar, c'est-à-dire avec -Ih-, dans un autre village du secteur, appelé Barkoxe, fr. Barcus. En outre, la phrase de Michelena n'est pas claire étant donné qu'on ne sait pas si cet auteur considère que cette forme Arachilar est la forme ancienne du nom Haritschelhar / Haritchelar ou si simplement Michelena cite, comme il le fait souvent au demeurant dans son ouvrage écrit au début des années cinquante, I'un à la suite de l'autre deux noms qu'il croyait probablement être apparentés bien que différents. Quoi qu'il en fût, l'auteur reconnaît que l'analyse de ces noms est difficile.

2 Tovar, 1987, p. 35.

3 Orpustan, 1999, p. 351.

4 Tovar, pp. 29-48.

5 Michelena, 1976, p. 335.

6 Rohlfs, 1970, p. 153, § 469.

7 Schomll, 1956, p. 311.

8 Tovar, 1987, p. 35. 
9 Information communiquée par Jacques Allières.

10 La forme Illibarits donnée en 1826 par les ingénieurs de la Marine n'est probablement pas une forme populaire, c'est-à-dire qu'il doit vraisemblablement s'agir d'une forme refaite par déduction logique ou par analogie avec d'autres formes commençant par $* \boldsymbol{I} \mathbf{I}$-. La meilleure explication reste néanmoins la suivante : les francophones ayant toujours eu beaucoup de difficulté à prononcer, encore de nos jours, des mots basques tels que iri (avec -r- faible), ils reproduisent d'ordinaire cette vibrante par $\boldsymbol{l}$. Il est en effet possible que les ingénieurs de la Marine, à n'en pas douter francophones, aient entendu *Iribarritz ou tout simplement Libarritz et qu'ils l'aient retranscrit par erreur IIlibarits.

11 Uranga, 1983, « Notas sobre topónimos... », p. 75.

12 Schuchartd, « Die Iberische... », p. 4

13 Menéndez Pidal, 1952, Toponimia... , p. 249, n. 19.

14 Lafon, 1958, « Noms de lieux d'aspect basque... », p. 130.

15 Caro Baroja l'expliquait à partir d'un cognomen non-attesté *Libarius, cf. supra.

16 Dauzat \& Rostaing, 1978, Dictionnaire étymologique... , p. 400, supposaient qu'il s'agissait d'un « nom d'homme lat. Liber, ou nom pré-latin, et suff. aquit. -ossum ». Rohlfs, 1952, p. 242, écrivait, quant à lui, « ce nom ne peut se séparer du cognomen Liber attesté en Espagne et en Gaule », ce qui ne paraissait pas convaincre René Lafon, «Noms de lieux d'aspect basque... », p. 130 , qui écrivait : « Mais le passage de e à a s'explique-t-il ? Il n'est donc pas sûr que Libarona et Libaros aient été tirés de mots latins. N'auraient-ils pas été tirés d'un mot pré-indo-européen Libar qui se serait conservé d'autre part sans suffixe dans le toponyme andalou ? ».

17 Michelena, 1989, Apellidos... , p. 106, § 332.

18 On pourra également comparer les toponymes Nabari(t)z (Anglet) / Navarosse (Biscarosse) $\leftrightarrow$ Libar(r)itz (Labourd) / Libaros (Hautes-Pyrénées). 
19 Afin de ce faire une idée sur cette question, on pourra consulter l'article suivant : Iglesias, 2000, «L'inscription de San Miguel de Liria... », pp. 7-27. 